\title{
A contribution to the braconid wasps (Hymenoptera: Braconidae) from Iran
}

\author{
Najmeh Samin ${ }^{1}$, Ahmet Beyarslan ${ }^{2}$, Juana María Coronado-Blanco ${ }^{3}$, \\ \& MAJID NAVAEIAN 4
}

\author{
${ }^{1}$ Young Researchers and Elite Club, Science and Research Branch, Islamic Azad University, \\ Tehran, Iran; email: n_samin63@yahoo.com \\ ${ }^{2}$ Department of Biology, Faculty of Arts and Science, Trakya University, \\ Edrine, Turkey \\ ${ }^{3}$ Facultad de Ingeniería y Ciencias, Universidad Autónoma de Tamaulipas, Centro Universitario, Cd. \\ Victoria, Tamaulipas, Mexico \\ ${ }^{4}$ Department of Biology, Yadegar-e Imam Khomeini (RAH) Shahre Rey Branch, \\ Islamic Azad University, Tehran, Iran
}

\begin{abstract}
Samin, N., Beyarslan, A., Coronado-Blanco, J.M., Navaeian, M. \& Kavallieratos, N.G.: A contribution to the braconid wasps (Hymenoptera: Braconidae) from Iran.

Abstract: In this faunistic paper, totally 24 species of Braconidae (Hymenoptera) were collected and identified from Alysiinae ( 5 species in 3 genera), Aphidiinae ( 2 species in 2 genera), Braconinae ( 4 species in 2 genera), Microgastrinae (4 species in one genus), Microgastrinae (3 species in 2 genera), Pambolinae (one species), Rhysipolinae (one species), Rhyssalinae (one species), and Rogadinae (3 species in 2 genera). Five species are new records for the fauna of Iran: Diaeretus leucopterus (Haliday, 1834) (Aphidiinae), Bracon (Glabrobracon) colpophorus Wesmael, 1838 (Braconinae), Apanteles (Dolichogenidea) punctiger (Wesmael, 1837) (Microgastrinae), Oncophanes (Oncophanes) minutus (Wesmael, 1838) (Rhyssalinae), and Clinocentrus caucasicus Tobias, 1976 (Rogadinae).
\end{abstract}

Keywords: Ichneumonoidea, Braconidae, parasitoids, hosts, fauna, new records, Iran

\section{Introduction}

Braconid wasps (Hymenoptera: Braconidae) with over than 21,000 described species worldwide (YU et al. 2016), are one of the most powerful parasitoids which have efficient role in biological control of different agricultural and forest pests (WAAGE \& Greathead 1986, Shaw \& Huddleston 1991, Wharton 1993, Quicke 2015). The fauna of Iranian Braconidae has been studied rather well (e.g., GADAllaH \& GHAHARI 2013a, 2013b, 2015, 2016, BARAHOEI et al. 2014, GADALLAH et al. 2015a, 2015b, 2016a, 2016b, FARAHANi et al. 2016, GHAHARi 2016, BEyARSLAn et al. 2017, SAMIN et al. 2018a, b), but since Iran is a large country comprises various ecosystems, several new species are expected to be discovered. The objective of this faunistic paper is to study of distribution of 24 braconid species in different regions and various ecosystems of Iran, and introducing of five new records for the fauna of Iran. 


\section{Material and methods}

The materials of this faunistic research were collected by Malaise traps and sweeping net from different regions and ecosystems of Iran. Some specimens were reared in optimum conditions in incubator $\left(25 \pm 2{ }^{\circ} \mathrm{C}, 65 \pm 5 \% \mathrm{RH}, 40: 14 \mathrm{~L}\right.$ : D). Additionally, some materials preserved in private collections, Natural History Museums, and insect collections of Universities were studied and used in this paper. Here we follow Yu et al. (2016) for nomenclature and classification.

\section{Results}

In total, 24 species of Braconidae within 8 subfamilies Alysiinae, Aphidiinae, Braconinae, Microgastrinae, Microgastrinae, Pambolinae, Rhysipolinae, Rhyssalinae, and Rogadinae have been collected and identified. The list of species is given below alphabetically.

Subfamily Alysiinae Leach, 1815

Aspilota ruficornis (Nees, 1834) - Ardebil province, Lahrood, 2§ðð, July 2016.

Chorebus (Chorebus) fordi (Nixon, 1954) - Ardebil province, Khalkhal, 29 , July 2016.

Chorebus (Chorebus) larides (Nixon, 1844) - East Azarbayjan province, Varzeghan, 2 우우, August 2015.

Dinotrema (Dinotrema) oleraceum (Tobias, 1962) - Kurdistan province, Qorveh, $1 \lesssim$, October 2013.

Dinotrema (Dinotrema) varipes (Tobias, 1962) - Lorestan province, Aligoodarz, $1 \widehat{\partial}$, June 2018.

\section{Subfamily Aphidiinae Haliday, 1833}

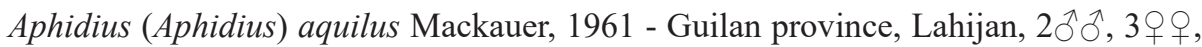
June 2013, ex Betulaphis quadrituberculata (Kaltenbach, 1843) (Homoptera, Aphididae).

Diaeretus leucopterus (Haliday, 1834) - West Azarbayjan province, Mian-Doab, $3 \widehat{\jmath} \widehat{\jmath}$, 5 우, July 2014, ex Schizaphis graminum (Rondani, 1852) (Hemiptera, Aphididae) on Hordeum vulgare L. (Poaceae).

\section{Subfamily Braconinae Nees, 1811}

Bracon (Glabrobracon) colpophorus Wesmael, 1838 - Ardebil province, Aslanduz, 3 우우, September 2014.

Bracon (Glabrobracon) pallicarpus Thomson, 1892 - Northern Khorasan province, Lujly, 2 우, May 2010.

Bracon (Glabrobracon) peroculatus (Wesmael, 1838) - Semnan province, Damghan, 1, 11.iv.2011; Razavi Khorasan province, Fariman, 1, April 2012.

Vipio simulator Kokujev, 1898 - West Azarbayjan province, Maku, 2 ㅇ , July 2012. 


\section{Subfamily Microgastrinae Förster, 1862}

Apanteles (Apanteles) erevanicus Tobias, 1976 - East Azarbayjan province, Horand, 2 우 , August 2008.

Apanteles (Dolichogenidea) drusilla Nixon, 1972 - Zanjan province province, Abhar, 1 , , September 2009.

Apanteles (Dolichogenidea) phaloniae Wilkinson, 1940 - Mazandaran province, Sari, $3 ㅇ ㅗ$, ex Zeuzera pyrina (Linnaeus, 1761) (Lepidoptera, Cossidae).

Apanteles (Dolichogenidea) punctiger (Wesmael, 1837) - Guilan province, Asalem, 4 우으, August 2014.

\section{Subfamily Microgastrinae Förster, 1862}

Cotesia melanoscela (Ratzeburg, 1844) - Golestan province, Golestan National Park, $3 ㅇ ㅜ$, October 2010, ex Euproctis chrysorrhoea (Linnaeus, 1758) (Lepidoptera, Erebidae).

Deuterixys carbonaria (Wesmael, 1837) - Guilan province, Talesh, 1ð, August 2013.

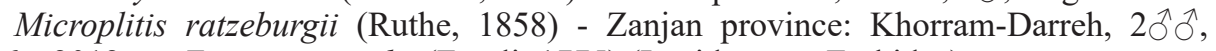
16.v.2012, ex Euproctis similis (Füssli, 1775) (Lepidoptera, Erebidae).

Subfamily Pambolinae Marshall, 1885

Phaenodus rugulosus Hellén, 1927 - West Azarbayjan province, Mahabad, $29+$, June 2012.

Subfamily Rhysipolinae Belokobylskij, 1984

Rhysipolis hariolator (Haliday, 1936) - Mazandaran province, Savadkooh (Ahangarkla), $2 \circ$ 우 $1{ }^{\widehat{O}}$, August 2014, ex Phyllonorycter salicicolella (Sircom, 1848) (Lepidoptera, Gracillariidae).

\section{Subfamily Rhyssalinae Förster, 1863}

Oncophanes (Oncophanes) minutus (Wesmael, 1838) - Guilan province, Lahijan,

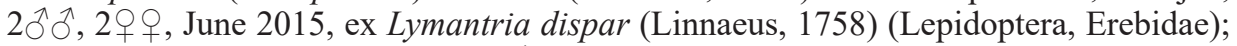
Mazandaran province, Behshahr, 1§̂, 2 우, July 2017, ex Archips rosana (Linnaeus, 1758) (Lepidoptera, Tortricidae).

\section{Subfamily Rogadinae Förster, 1863}

Aleiodes (Neorhogas) caucasicus (Tobias, 1976) - Ardebil province, Aslanduz, 1ठ઼, September 2016.

Aleiodes (Aleiodes) signatus (Nees, 1811) - Golestan province, Galikesh (Sarcheshmeh), 3 우, June 2009, ex Orgyia antiqua (Linnaeus, 1758) (Lepidoptera: Erebidae).

Clinocentrus caucasicus Tobias, 1976 - Zanjan province, Khorram-Darreh, 2 $q$, July 2015. 


\section{Acknowledgements}

The authors are grateful to Maximilian Fischer (Austria), Kees van Achterberg (Netherlands), and the late J. Papp (Hungary) for their help in this project. This research was supported by Islamic Azad University, Universidad Autónoma de Tamaulipas, and Trakya University.

\section{References}

Barahoei, H., Rakhshani, E., Nader, E., Starý, P., Kavallieratos, N.G., Tomanović, Z. \& Mehrparvar, M. 2014: Checklist of Aphidiinae parasitoids (Hymenoptera: Braconidae) and their host aphid associations in Iran. - Journal of Crop Protection 3(2): 199-232.

Beyarslan, A., Gadallah, N.S. \& Ghahari, H. 2017: An annotated catalogue of the Iranian Microtypinae and Rogadinae (Hymenoptera: Braconidae). - Zootaxa 4291(1): 099-116. DOI: https://doi.org/10.11646/ zootaxa.4291.1.6

Farahani, S., TAlebi, A.A. \& Rakhshani, E. 2016: Iranian Braconidae (Insecta: Hymenoptera: Ichneumonoidea): diversity, distribution and host association. - Journal of Insect Biodiversity and Systematics 2(1): 1-92.

Gadallah, N.S. \& Ghahari, H. 2013a: An annotated catalogue of the Iranian Agathidinae and Brachistinae (Hymenoptera: Braconidae). - Linzer biologische Beiträge 45/2: 1873-1901.

Gadallah, N.S. \& GHahari, H. 2013b: An annotated catalogue of the Iranian Cheloninae (Hymenoptera: Braconidae). - Linzer biologische Beiträge 45/2: 1921-1943.

Gadallah, N.S. \& Ghahari, H. 2015: An annotated catalogue of the Iranian Braconinae (Hymenoptera: Braconidae). - Entomofauna 36: 121-176.

Gadallah, N.S., Ghahari, H., Peris-Felipo, F. J. \& Fischer, M. 2015a: An annotated catalogue of the Iranian Alysiinae (Hymenoptera: Braconidae). - Zootaxa 3974(1): 1-28. DOI: https://doi.org/10.11646/ zootaxa.3974.1.1

Gadallah, N.S., Ghahari, H. \& Peris-Felipo, F. J. 2015b: Catalogue of the Iranian Microgastrinae (Hymenoptera: Braconidae). - Zootaxa 4043(1): 1-69. DOI: http://dx.doi.org/10.11646/zootaxa.4043.1.1

Gadallah, N.S., Ghahari, H., Peris-Felipo, F.J. \& Fischer, M. 2016a: Updated checklist of Iranian Opiinae (Hymenoptera: Braconidae). - Zootaxa 4066(1): 1-40. DOI: http://doi.org/10.11646/zootaxa.4066.1.1

Gadallah, N.S., Ghahari, H. \& van AchterberG, C. 2016b: An annotated catalogue of the Iranian Euphorinae, Gnamptodontinae, Helconinae, Hormiinae and Rhysipolinae (Hymenoptera: Braconidae). Zootaxa 4072(1): 1-38.

Gadallah, N.S. \& Ghahari, H. 2016: An updated checklist of the Iranian Miracinae, Pambolinae and Sigalphinae (Hymenoptera: Braconidae). - Orsis 30: 51-61.

GadAllah, N.S. \& GHAhARI, H. 2017: An annotated catalogue of the Iranian Doryctinae and Exothecinae (Hymenoptera: Braconidae). - Transactions of the American Entomological Society 143: 669-691. DOI: https://doi.org/10.3157/061.143.0308

GHAHARI, H. 2016: Five new records of Iranian Braconidae (Hymenoptera: Ichnemonoidea) for Iran and annotated catalogue of the subfamily Homolobinae. - Wuyi Science Journal 32: 35-43.

Quicke, D.L.J. 2015: The braconid and ichneumonid parasitoid wasps: Biology, systematics, evolution and ecology. - Wiley Blackwell, Chichester, 688 pp.

Samin, N., Coronado-Blanco, J.M., Kavallieratos, N.G., Fischer, M., \& SaKenin, H. 2018a: Recent findings on Braconidae (Hymenoptera: Ichneumonoidea) of Iran with an updated checklist. - Acta Biologica Turcica 31(4): 160-173.

Samin, N., Coronado-Blanco, J.M., Fischer, M., van Achterberg, C., Sakenin, H. \& Davidian, E. 2018b: Updated checklist of Iranian Braconidae (Hymenoptera: Ichneumonoidea) with twenty-three new records. - Natura Somogyiensis 32: 21-36. DOI: https://doi.org/10.24394/NatSom.2018.32.21

Shaw, M.R. \& Huddleston, T. 1991: Classification and biology of braconid wasps (Hymenoptera: Braconidae). Royal Entomological Society of London. - Handbooks for the Identification of British Insects, London, $126 \mathrm{pp}$.

WaAge, J. \& Greathead, D. 1986: Insect parasitoids. Acadamic Press, London, xviii + 389 pp.

Wharton, R.A. 1993: Bionomics of the Braconidae. - Annual Review of Entomology 38: 121-143. DOI: https://doi.org/10.1146/annurev.en.38.010193.001005

Yu, D.S., van Achterberg, K. \& Horstmann, K. 2016: World Ichneumonoidea 2011. - Taxonomy, Biology, Morphology and Distribution. Taxapad.com. Canada. 